\title{
Contextual specificity of extinction of delay but not trace eyeblink conditioning in humans
}

\author{
Christian Grillon, ${ }^{1}$ Ruben P. Alvarez, Linda Johnson, and Chanen Chavis \\ Mood \& Anxiety Disorders Program, National Institute of Mental Health, NIH, Bethesda, Maryland 20892, USA
}

\begin{abstract}
Renewal of an extinguished conditioned response has been demonstrated in humans and in animals using various types of procedures, except renewal of motor learning such as eyeblink conditioning. We tested renewal of delay and trace eyeblink conditioning in a virtual environment in an $A B A$ design. Following acquisition in one context $(A$, e.g., an airport) and extinction in a different context (B, e.g., a city), tests for renewal took place in the acquisition (A) and extinction context (B), in a counterbalanced order. Results showed renewal of the extinguished conditioned response in the delay but not trace condition.
\end{abstract}

Contexts play an important role in the learning and expression of conditioned responses (CRs). In conditioning experiments, the preponderant view is that, while CRs following training generalize across contexts, extinguished CRs are context-specific (Bouton 2004). The context dependence of extinction has been demonstrated in renewal experiments in which, following acquisition training in one context (A), extinction training takes place in a different context (B). Extinction performance is then tested in a context different from the extinction context. Under these conditions, fear reemerges or renews following a return to the original acquisition context (ABA design) (Bouton 2004).

Renewal is a robust effect, which has been documented in animals in appetitive and aversive conditioning as well as in flavor aversion learning (see Bouton 2002). Recently, renewal has also been demonstrated in humans using various aversive conditioning procedures and various types of CRs (Vansteenwegen et al. 2005; Alvarez et al. 2007). These results confirm that extinction of aversive conditioning is highly sensitive to contextual information while conditioned fear is not. However, less is known about contextual influence on motor learning such as eyeblink conditioning (EBC) in humans and in animals.

There is some evidence that the context does not influence $\mathrm{EBC}$ as it does other forms of CR in rodents. For example, Penick and Solomon (1991) showed that, following conditioning of the nictitating membrane with a puff, a switch in context disrupted substantially the expression of the CR (Penick and Solomon 1991). However, a subsequent study did not replicate these results (Lindquist and Brown 2004). Another study found that the expression of CRs was substantially reduced after postacquisition exposure to the acquisition context relative to a home-cage control condition (Poulos et al. 2006). So far, we are not aware of any study that has investigated renewal of an extinguished CR in an EBC paradigm in humans or in animals. The main aims of this study were to examine whether EBC generalized across contexts and renewed in an ABA design in humans.

During EBC, puff of air to the eye (US: unconditioned stimulus) is paired repeatedly with a short-duration tone (CS: conditioned stimulus), causing subjects to blink (unconditioned response). Following several tone-puff pairings, a conditioned eyeblink response develops subsequent to the tone presentation and prior to the delivery of the puff. EBC can be examined during delay or trace paradigms. In delay EBC, the puff is administered

1'Corresponding author.

E-mail christian.grillon@nih.gov; fax (301) 594-9959.

Article is online at http://www.learnmem.org/cgi/doi/10.1101//m.855708. at the end of the tone. Trace EBC differs from delay EBC in that the puff follows an empty (trace) interval (e.g., $800 \mathrm{msec}$ ) separating the tone from the onset of the puff. Trace EBC is more complex than delay EBC because of the temporal gap between the conditioned stimulus and the unconditioned stimulus, which requires learning about discontinuous events in time before learning can occur. The hippocampus is critical for trace EBC but not for delay EBC (Moyer et al. 1990; Christian and Thompson 2003). Clinical studies indicate reduced trace EBC in patients with temporal lesions with trace intervals that exceed $1000 \mathrm{msec}$ (Woodruff-Pak 1993; McGlinchey-Berroth et al. 1997; Clark and Squire 1998). Delay and trace EBC also differ in that the delay condition is considered an implicit learning task that can be conducted outside a subject's awareness of the tone-puff contingency, whereas the trace condition is an explicit learning task that requires awareness of the tone-puff contingency (Clark and Squire 1998). Because trace EBC requires more cognitive resources than delay $\mathrm{EBC}$, it is possible that context shifts are more likely to affect trace than delay EBC, especially following extinction when the conditioned stimulus becomes ambiguous. We predicted normal generalization and renewal of EBC in the delay condition, but did not make any prediction regarding trace EBC because of the possibility the shift in contexts between conditions (extinction and renewal) interferes with performance. We recently reported robust renewal of fear-potentiated startle in a delay fear conditioning paradigm using computer-generated virtual reality (VR) (Alvarez et al. 2007). We used the same virtual environment in the present study.

There were 13 (mean age $26.6 \mathrm{yr}$; SD = $4.7 \mathrm{yr}$; eight females) and 15 (mean age $26.8 \mathrm{yr} ; \mathrm{SD}=5.1 \mathrm{yr}$; nine females) healthy volunteers in the delay and trace groups, respectively, who gave written informed consent. Each virtual environment, an airport and a city, was prerecorded and rendered as digitized scenes, which subjects passively observed on a large screen $(50 \times 35 \mathrm{in}$. $)$ (Alvarez et al. 2007). Subjects underwent conditioning in one context (A) and extinction in another (B), after which renewal was tested in two contexts: the acquisition context (A) and the extinction context (B) (counterbalanced across subjects). In six subjects in the delay group and seven subjects in the trace group, the airport and the city served as acquisition and extinction contexts, respectively, with the order reversed in the remaining subjects.

Due to the long duration of the test, three recording phases separated by 5 -min breaks were implemented. Phase 1 was the first acquisition period (lasting $694 \mathrm{sec}$ ). Phase 2 consisted of a second acquisition period (490 sec) and the first extinction period $(250 \mathrm{sec})$. Phase 3 included a second extinction period (250 
sec) and the two renewal tests (each lasting $55 \mathrm{sec}$ ). It took $30 \mathrm{sec}$ to be "moved" from on context to another via a street and a subway. There were nine acquisition blocks and four extinction blocks of 10 trials per block. Each acquisition block contained nine CS-puffs and one unreinforced CS and each extinction block contained 10 unreinforced CS.

Phase 1 started with the delivery of four unreinforced CS followed by four puffs-alone. This was followed by five acquisition blocks. Phase 2 contained four additional acquisition blocks and two extinction blocks. Phase 3 started with two additional extinction blocks, followed by the two renewal tests. During renewal, participants visited the acquisition and extinction contexts once each in a counterbalanced order. During each visit, they received four presentations of the CS alone. The time interval between CS varied from 10 to $14 \mathrm{sec}$. After a context change, the first CS was presented 9-12 sec after entry in the new context.

The tone (CS) was a 500-msec duration, pure $1 \mathrm{kHz}$ tone at $75 \mathrm{~dB}(\mathrm{~A})$ delivered binaurally through headphones. The 50-msec duration US was a 10-psi air puff (measured at the level of the tank of compressed air) delivered to the left cornea via a 5 -ft tubing attached to customized headgear. In the delay group, the puff co-terminated with the CS. In the trace group, the puff was delivered $1000 \mathrm{msec}$ after CS onset (500-msec trace interval). The eyeblinks were recorded with two miniature electrodes placed under the left eye with a ground placed on the forehead. Electrode impedance was below $5 \mathrm{k} \Omega$. The EMG was filtered (30-500 $\mathrm{Hz}$ ) and integrated with a 10-msec time constant.

An eyeblink was considered a CR if it reached at least $4 \mathrm{mV}$ and occurred in a 400-msec window, $100-500$ msec post-CS onset in the delay group, and $600-1000 \mathrm{msec}$ in the trace group. CRs during acquisition and extinction were calculated as the percentage of eyeblink responses in blocks of 10 trials. CRs during renewal were calculated as the percentage of eyeblink responses within each context. We estimated the percentage of spontaneous eyeblinks in a similar way by calculating the number of eyeblinks that were $>4 \mathrm{mV}$ in the $400 \mathrm{msec}$ preceding each CS. This provided a measure against which to compare CR during acquisition. Preliminary analyses indicated that there were very few spontaneous eyeblinks during the 100-500 msec post-CS onset in the trace group, showing no increase over trials during acquisition. The results of these analyses are not reported.

Statistical analyses of CR were conducted with analyses of variance (ANOVAs) with repeated measures. The CR during acquisition was entered in a Group (delay, trace) $\times$ Eyeblink Type $(\mathrm{CR}$, spontaneous eyeblinks) $\times$ Block (nine blocks) ANOVA. The comparison between $\mathrm{CR}$ and spontaneous eyeblinks was not tested in subsequent analyses because (1) there were very few spontaneous eyeblinks in both groups and (2) the presence of cells with a 0 score prevented the use of ANOVAs. Generalization across the acquisition and extinction context was examined by comparing CR during the last acquisition block to CR during each of the first five extinction trials in Group (delay, trace) $\times$ Trials (six data points) ANOVA. The extinction CR was entered in a Group (delay, trace) $\times$ Block (four blocks for extinction) ANOVA. The order of contexts was not considered in the statistical analysis because preliminary analyses did not show any effect on this variable. The percent CR during renewal was analyzed with a Group (delay, trace) $\times$ Context (acquisition, extinction) ANOVA. This analysis was conducted with the first trial in each context as well as with the average of the four trials per context. Greenhouse-Geisser corrections $(G G-\varepsilon)$ were used when required.

Figure 1 shows the percent CR and percent spontaneous eyeblinks in the delay and trace groups during acquisition and extinction. Successful conditioning was observed as reflected by a greater percent CR compared to percent spontaneous eyeblinks during acquisition (Eyeblink Type: $F_{(1,26)}=59.5, P<0.0001$ ) as well as an Eyeblink Type $\times$ Block interaction $\left(F_{(8,208)}=3.7\right.$, $P<0.002)$ and a linear Eyeblink Type $\times$ Block interaction $\left(F_{(1,26)}=7.5, P<0.01\right)$. There was no significant difference between the two groups.

CRs generalized across the acquisition and extinction contexts but extinguished rapidly (Trial main effect: $F_{(1,130)}=3.22$, $P<0.01, \varepsilon=0.87)$. The percent CR during the last acquisition block (mean $=0.43$, SEM $=0.05$ ) did not differ from CR during the first (mean $=0.46, \mathrm{SEM}=0.09 ; F_{(1,26)}=0.12$, not significant $[\mathrm{ns}]$ ) and third (mean $=0.30, \mathrm{SEM}=0.09 ; F_{(1,26)}=2.3$, ns) extinction trials but was significantly higher than during trials 2 $($ mean $=0.29, \mathrm{SEM}=0.08), 4($ mean $=0.14, \mathrm{SEM}=0.06)$, and 5 (mean $=0.18$, SEM $=0.06)($ all $P>0.05)$.

The percent CR diminished progressively during extinction (linear Eyeblink Type $\times$ Block interaction: $F_{(1,26)}=12.1$, $P<0.002)$. Again, there was no group difference.

Figure 2 shows the renewal effect in each context. The delay group but not the trace group showed the expected renewal effect. Differential renewal in the two groups was present based on the first trial in each context (Group $\times$ Context: $F_{(1,26)}=16.2$, $P<0.002$ ) and based on the average of the four trials (Group $\times$ Context: $\left.F_{(1,26)}=12.9, P<0.002\right)$. The delay group showed greater percent $\mathrm{CR}$ in the acquisition context compared to the extinction context $\left(F_{(1,26)}=16.3, P<0.0009\right)$. The trace group showed no difference in percent $\mathrm{CR}$ between the two contexts $\left(F_{(1,26)}=0.88\right)$. In addition, the percent CR was higher in the acquisition context during renewal than in the last block of extinction in the delay group $\left(F_{(1,26)}=17.3, P<0.0009\right)$ but not in the trace group $\left(F_{(1,26)}=0.34\right)$. An additional analysis restricted to the trace group examined renewal in subjects who went in the acquisition context first followed by the extinction context and in subjects who went to the extinction context first followed by the acquisition context during renewal. Results remain the same. There was no evidence of renewal in any group.

The main results of this study were that (1) delay and trace EBC generalized across contexts and (2) delay EBC showed the traditional renewal effect following extinction, but trace EBC did not. Following extinction, delay EBC renewed when perfor-

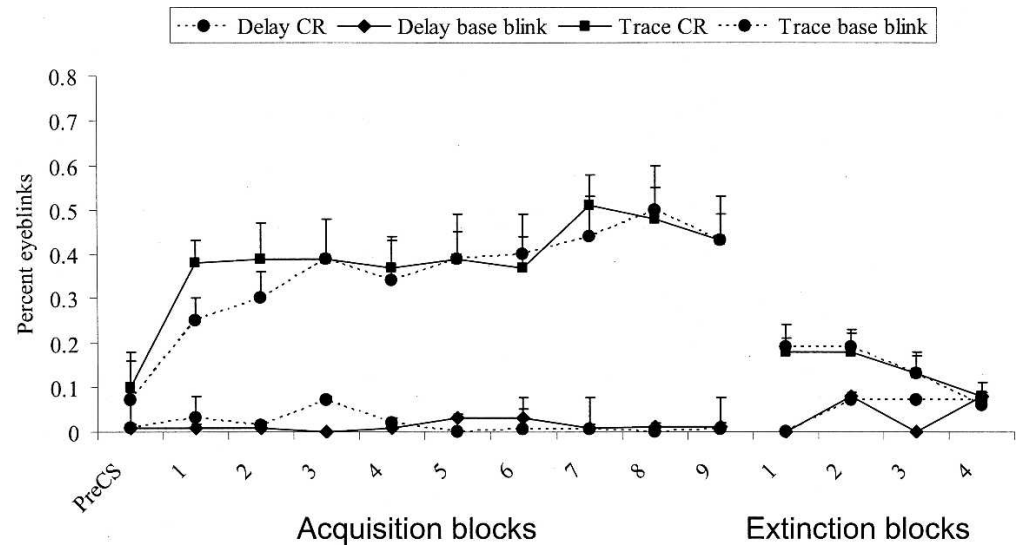

Figure 1. Percent $\mathrm{CR}$ and spontaneous eyeblinks during acquisition and extinction in the delay and trace $E B C$ groups. Error bars are standard errors. 


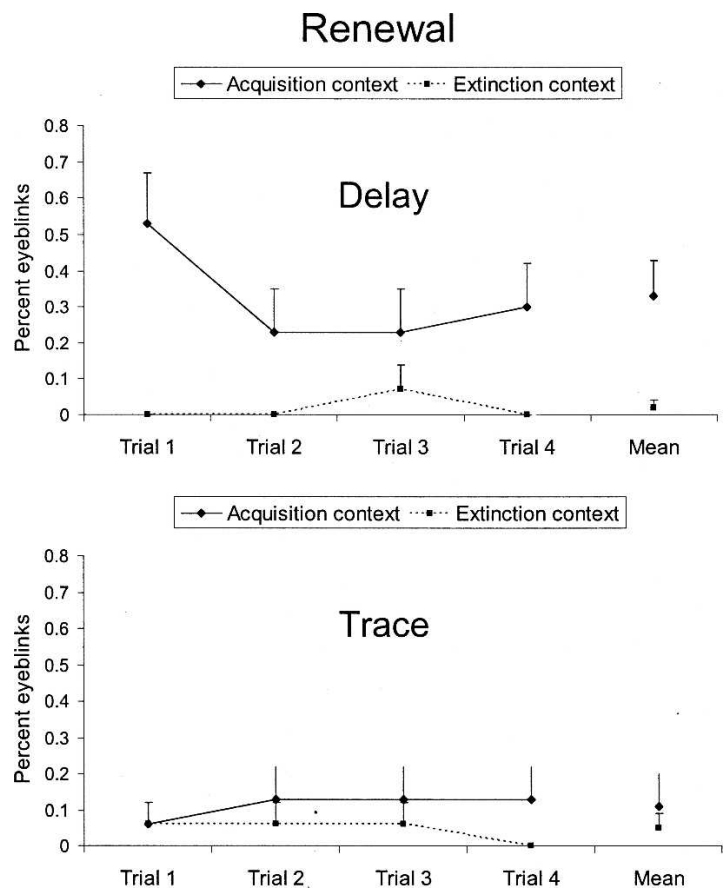

Figure 2. Percent $C R$ during renewal in the extinction and acquisition contexts in the delay and trace EBC groups. Error bars are standard errors. Results are shown for each trial and for the mean percent CR over the four trials.

mance was tested in a context other than the extinction context (i.e., the acquisition context). The percent delay EBC was greater in the renewal acquisition context compared to both the renewal extinction context and the last block of extinction. Trace EBC showed no renewal effect. The percent trace EBC did not differ significantly among the renewal acquisition context, the renewal extinction context, and the last block of extinction.

This is the first study documenting generalization across contexts and renewal following extinction of EBC in humans. We are also not aware of any study that has examined renewal of EBC in animals. The generalization data are consistent with the literature. Excitatory conditioning is known to generalize across contexts (Bouton 2004). However, there was a rapid decline in $\mathrm{CR}$ at the beginning of extinction that may either be the consequence of rapid extinction or the result of context shift. Future studies should include a "no context change" control group to address this issue.

The renewal of delay EBC is also consistent with the literature. The reemergence of extinguished CRs has been demonstrated using various procedures, including aversive and appetitive conditioning. The present study adds to the literature in showing that motor learning can also show renewal.

There is no clear explanation for the lack of renewal in the trace condition. Delay and trace conditioning paradigms differed in two crucial points. First, there was a temporal gap between the CS and the US during trace, but not delay conditioning. Second, the CS onset-US onset time interval was longer in the trace compared to the delay condition, making trace EBC a potentially more difficult task. The role of the hippocampus in long-delay EBC is not clear. The hippocampus appears to be critical for acquiring long-delay EBC in rodents (Beylin et al. 2001) but not in humans (Clark and Squire 1998). The lack of renewal effect may reflect the difficulty or fragility of trace EBC. However, there was no empirical support for stronger conditioning in the delay group compared to the trace group. Performance was similar dur- ing delay and trace conditioning during acquisition and extinction, suggesting no group difference in strength of conditioning. It is possible that performance during trace EBC was artificially inflated by mistakenly counting spontaneous eyeblinks as CR. This is unlikely because the percent CR was estimated based on a similar (400-msec) window of analysis for the delay and trace condition. In addition, the percent spontaneous eyeblinks did not differ in the two conditions.

The lack of renewal in the trace group could be due to cognitive factors. Delay and trace EBC are considered implicit and explicit learning tasks, respectively (Clark and Squire 1998, 1999). While both types of EBC are dependent on the cerebellum, trace EBC differs from delay EBC in that it requires awareness of stimulus contingency between the CS and the US (Clark and Squire 1998). Trace EBC is easily disrupted by external inhibition. For example, trace EBC, but not delay EBC, is impaired when subjects are engaged in a secondary task such as watching a movie (Clark and Squire 1999). It is possible that the renewal context, by drawing attention away from attentional resources, interfered with the performance of trace EBC.

To summarize, this study reports that motor learning, as measured by delay EBC, can also renew following extinction. Since trace EBC involves conscious processing of the CS, the failure of the trace group to show a renewal effect may have been caused by reduced attention to the CS due to the context shift during renewal.

\section{Acknowledgments}

This research was supported by the Intramural Research Program of the National Institute of Mental Health.

\section{References}

Alvarez, R.P., Johnson, L., and Grillon, C. 2007. Contextual-specificity of short-delay extinction in humans: Renewal of fear-potentiated startle in a virtual environment. Learn. Mem. 14: 247-253.

Beylin, A.V., Gandhi, C.C., Wood, G.E., Talk, A.C., Matzel, L.D., and Shors, T.J. 2001. The role of the hippocampus in trace conditioning: Temporal discontinuity or task difficulty? Neurobiol. Learn. Mem. 76: $447-461$.

Bouton, M.E. 2002. Context, ambiguity, and unlearning: Source of relapse after behavbioral extinction. Biol. Psychiatry 52: 976-986.

Bouton, M.E. 2004. Context and behavioral processes in extinction. Learn. Mem. 11: 485-494.

Christian, K.M. and Thompson, R.F. 2003. Neural substrates of eyeblink conditioning: Acquisition and retention. Learn. Mem. 10: 427-455.

Clark, R.E. and Squire, L.R. 1998. Classical conditioning and brain systems: The role of awareness. Science 280: 77-81.

Clark, C.R. and Squire, L.R. 1999. Human eyeblink classical conditioning: Effects of manipulating awareness of the stimulus contingencies. Psychol. Sci. 10: 14-18.

Lindquist, D.H. and Brown, T.H. 2004. Amygdalar NMDA receptors control the expression of associative reflex facilitation and three other conditional responses. Behav. Neurosci. 118: 36-52.

McGlinchey-Berroth, R., Carrillo, M.C., Gabrieli, J.D., Brawn, C.M., and Disterhoft, J.F. 1997. Impaired trace eyeblink conditioning in bilateral, medial-temporal lobe amnesia. Behav. Neurosci. 111: $873-882$.

Moyer Jr., J.R., Deyo, R.A., and Disterhoft, J.F. 1990. Hippocampectomy disrupts trace eye-blink conditioning in rabbits. Behav. Neurosci. 104: $243-252$.

Penick, S. and Solomon, P.R. 1991. Hippocampus, context, and conditioning. Behav. Neurosci. 105: 611-617.

Poulos, A.M., Pakaprot, N., Mahdi, B., Kehoe, E.J., and Thompson, R.F. 2006. Decremental effects of context exposure following delay eyeblink conditioning in rabbits. Behav. Neurosci. 120: 730-734.

Vansteenwegen, D., Hermans, D., Vervliet, B., Francken, G., Beckers, T., Baeyens, F., and Eelen, P. 2005. Return of fear in a human differential conditioning paradigm caused by a return to the original acquistion context. Behav. Res. Ther. 43: 323-336.

Woodruff-Pak, D. 1993. Eyeblink classical conditioning in H.M.: Delay and trace paradigms. Behav. Neurosci. 107: 911-925.

Received November 23, 2007; accepted in revised form March 24, 2008. 


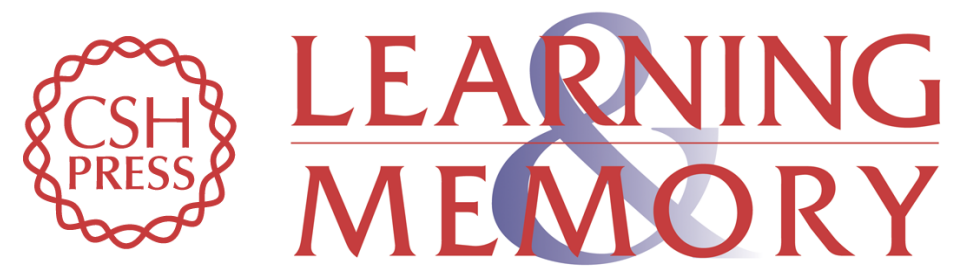

\section{Contextual specificity of extinction of delay but not trace eyeblink conditioning in humans}

Christian Grillon, Ruben P. Alvarez, Linda Johnson, et al.

Learn. Mem. 2008, 15:

Access the most recent version at doi:10.1101//m.855708

References This article cites 14 articles, 4 of which can be accessed free at:

http://learnmem.cshlp.org/content/15/6/387.full.html\#ref-list-1

License

Email Alerting Receive free email alerts when new articles cite this article - sign up in the box at the Service top right corner of the article or click here. 